\title{
Investigating the Effect of Hyperlink Information Scent on Users' Interaction with a Web Site
}

\author{
Nikolaos Tselios, Christos Katsanos, and Nikolaos Avouris \\ Human-Computer Interaction Group, Department of Electrical and Computer Engineering, \\ University of Patras, GR-265 00 Rio Patras, Greece \\ \{nitse, ckatsanos\}@ece.upatras.gr, avouris@upatras.gr
}

\begin{abstract}
In the study presented in this paper we investigate how variations of information scent of hyperlinks of a webpage influence users' behavior in terms of attention-focusing, confidence, effectiveness and efficiency, while exploring a website. In the reported study, 19 participants completed eight different tasks associated with eight simplified websites. Analysis of the results showed that even small differences in the target-link's information scent can substantially affect users' performance, distribution of attention and confidence. The study contributes to the related literature by quantifying the impact of even small differences in the target-link's scent on users' success ratio, time for first click, confidence and distribution of attention. In addition, a scent threshold value was identified, below which all the measured variables were substantially affected, and thus the link could be characterized as of "weak scent".
\end{abstract}

Keywords: Information scent, eye tracking study, Latent Semantic Analysis.

\section{Introduction}

Information scent has been defined as the users' assessment of the value of following a particular hyperlink in a webpage, based on its perceived semantic association with their goal [1], [2], [3], [7]. Users have lower success rates and require more time to complete their tasks when they are presented with navigation options of weak information scent compared to high information scent [2], [3], [7].

Thus, systematic study of the impact of scent variations on users' performance is important. Such quantitative studies are also needed to identify the threshold value over which one can characterize a link as of high scent, which is often determined in an arbitrary way currently. Furthermore, eye tracking user studies, such as [4] and the one reported in this paper, can provide insights on the effect of information scent on users' distribution of attention while selecting links in a webpage.

In the eye-tracking user study presented in this paper, we investigated the impact of slight variations of the target-link's information scent on users' effectiveness, efficiency, confidence and attention-focusing while selecting a link in a webpage. The paper is organized as follows: First, the method of the study is presented, followed by the analysis of results. Finally, we discuss the conclusions, implications and future directions of the presented research. 


\section{Method of the Study}

Eight webpages were built that contained navigation menus of actual websites, related to specific tasks (e.g., find a destination, buy a specific object, etc.) ${ }^{1}$. The textual descriptions of the tasks for these pages were produced in a way that created eight levels of the correct-link's information scent, measured using an estimation of semantic similarity between the correct-link's text and the description of the task.

This estimation was provided by Latent Semantic Analysis (LSA, [6]), a technique that relies on the statistical elaboration of corpora to estimate the semantic similarity between two texts. Overall, the LSA index of the correct-links of the eight pages ranged from 0.7 to 0 . The rest of the hyperlinks had LSA values below 0.15 (Table1). Two experts in information architecture provided judgments of the information scent of all links, as a means of preliminary evaluation of the validity of LSA in the context of the given tasks. Miller and Remington [8] involved three judges to assess the information scent of hyperlinks in their experimental webpages. Thus, two judges were deemed enough to evaluate the validity of LSA in our case.

Subsequently, 19 participants, 5 female, with a mean age of 24 , all proficient in English (the language of the webpages), were asked to perform the eight menu selection tasks using a typical Web browser. Their behavior was monitored. All navigation menus were presented in a single-column format and included eight link-options. The order of presentation of the websites and the order of links in the homepages were randomized to avoid serial order effects. First, users were presented with a goaldescription screen. Next, they were presented with the associated menu and were asked to select a link as they would normally do. Afterwards, they subjectively rated how confident they felt for their selection on a 1-10 scale. An unobtrusive 17' Tobii T60 eye tracker with minimum fixation duration set to $100 \mathrm{~ms}$ was used to record users' eye movements during this process.

Four measures of users' behavior were gathered: a) Effectiveness = correct firstclick percentage, b) Efficiency = time for first-click, c) Confidence = users'

Table 1. Users' metrics of link-selection behavior collected

\begin{tabular}{|c|c|c|c|c|c|c|c|c|c|}
\hline \multirow{2}{*}{$\operatorname{Task}^{1}$} & \multirow{2}{*}{$\begin{array}{c}\text { Correct- } \\
\text { link } \\
\text { Scent }\end{array}$} & \multirow{2}{*}{$\begin{array}{c}2^{\text {nd }} \text {-best } \\
\text { link } \\
\text { Scent }\end{array}$} & \multirow{2}{*}{$\begin{array}{c}\text { Success } \\
(\%)\end{array}$} & \multirow{2}{*}{$\begin{array}{l}\text { Click } \\
\text { time } \\
(\mathrm{sec})\end{array}$} & \multirow{2}{*}{$\begin{array}{c}\text { Confidence } \\
(1-10)\end{array}$} & \multicolumn{2}{|c|}{$\begin{array}{c}\text { Number of } \\
\text { observations }\end{array}$} & \multicolumn{2}{|c|}{$\begin{array}{c}\text { Observations } \\
\text { Duration (sec) }\end{array}$} \\
\hline & & & & & & $\begin{array}{c}\text { Correct } \\
\text { link }\end{array}$ & $\begin{array}{c}\text { All } \\
\text { links }\end{array}$ & $\begin{array}{c}\text { Correct } \\
\text { link }\end{array}$ & $\begin{array}{c}\text { All } \\
\text { links }\end{array}$ \\
\hline 1 & 0.70 & 0.09 & $89 \%$ & 12 & 8.9 & 2.5 & 13.8 & 2.3 & 11.0 \\
\hline 2 & 0.59 & 0.14 & $74 \%$ & 21 & 8.1 & 3.6 & 18.1 & 5.2 & 19.7 \\
\hline 3 & 0.50 & 0.10 & $68 \%$ & 19 & 7.9 & 1.9 & 15.9 & 2.6 & 16.5 \\
\hline 4 & 0.40 & 0.01 & $68 \%$ & 17 & 6.8 & 3.0 & 19.3 & 2.4 & 15.8 \\
\hline 5 & 0.30 & 0.11 & $68 \%$ & 14 & 7.5 & 3.4 & 17.3 & 3.0 & 14.6 \\
\hline 6 & 0.21 & 0.06 & $37 \%$ & 28 & 6.3 & 3.9 & 24.2 & 3.7 & 27.0 \\
\hline 7 & 0.11 & 0.08 & $21 \%$ & 22 & 6.3 & 3.4 & 19.6 & 4.3 & 19.5 \\
\hline 8 & -0.01 & 0.08 & $11 \%$ & 31 & 6.1 & 4.2 & 26.6 & 4.7 & 30.0 \\
\hline
\end{tabular}

\footnotetext{
${ }^{1}$ Tasks and menus used can be found at http://hci.ece.upatras.gr/Tselios_et_al_INT2009.
} 
confidence for first-click, d) Efficiency of visual search measured in terms of number of observations on each link and duration of observations on each link.

\section{Analysis and Results}

The values of users' effectiveness, efficiency, confidence and visual search efficiency for each task, as measured by the selected dependent variables are presented in Table 1. Scent values of the correct link and the link with the highest scent value among the rest are also shown.

Correlation analysis indicated a very high degree of correlation between the scent value of the correct link and the observed participants' success ratio (Fig. 1a, r=0.945, $\mathrm{p}<.001$ ), average time for click (Fig. $1 \mathrm{~b}, \mathrm{r}=-0.725, \mathrm{p}<0.05$ ), subjective average confidence (Fig. 1c, r=0.934, p<0.01), and number of observations on all links $(r=-0.839$, $\mathrm{p}<0.05)$. A trend was also observed between the scent value of the correct link and the average number $(\mathrm{r}=-0.688$, ns) and duration $(\mathrm{r}=-0.436$, $\mathrm{ns})$ of observations on the correct link. Furthermore, there seems to be a critical scent value for the correct link between 0.21 and 0.30 . Below this value, the measured variables appear to be substantially affected and therefore, the link can be characterized as of weak scent.

Fig. 1d presents heatmaps of participants' total duration of fixations in each webpage. In higher scent pages, attention was mainly focused in the area containing the
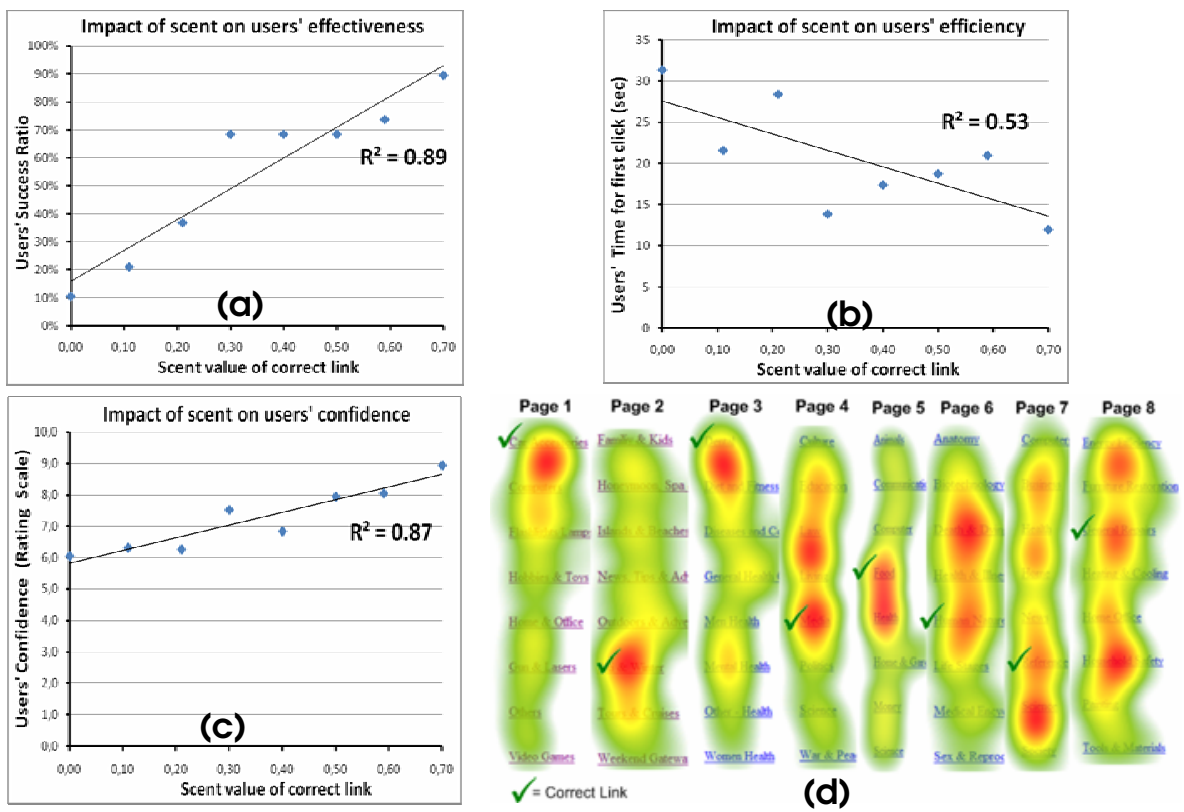

Fig. 1. Scatter plots of the scent values of the correct link for each task and the observed participants' success ratio (a), average click time (b), and average confidence (c). Heatmaps of participants' total duration of fixations for each webpage (d). 
correct link, indicating a focused and efficient search. In webpages 4-5 there is a slight distribution of attention across some other links indicating a potential lack of confidence regarding the correct choice, which can be also observed in the users' average self-reported confidence (see Table 1). In webpages 6 to 8 , in which the scent value of the correct link dropped substantially, the attention was distributed across the majority of links indicating increased confusion. In these three tasks, users' success ratio was on average $23 \%$, whereas in the rest five it was $73 \%$.

\section{Conclusions}

The results of an eye-tracking study are reported in which the impact of eight, slightly different levels of information scent was measured on various aspects of users' linkselection behavior. When scent increased, users were gradually getting more effective and efficient, had significantly more focused attention-allocation patterns (also reported in [4]) and reported higher levels of selection confidence. It was observed that even small differences in the target-link's scent could substantially affect the collected measures of users' behavior. For instance, link selection time in task 1 was 12 seconds, while in task 2 was 21 seconds, with a target-link's scent value of 0.70 and 0.59 respectively. Pirolli [7] also found high correlation $(\mathrm{r}=0.949)$ between the observed and theoretically predicted (generated by Monte Carlo simulations using an ACTScent model) frequency of users' link-selections, very close to our results.

In addition, it was found that the LSA threshold above which a link could be characterized as of high scent is between 0.21 and 0.30 , also empirically reported in [1]. Such identification of threshold levels could significantly extend the utility of automated evaluation tools, such as ACWW [1] and ISEtool [5].

The findings of this study may be extended and further validated by extensive testing with a richer set of tasks that take into account other influencing factors (e.g. layout, number of available links), and other methods to compute information scent (such as human rating, PMI-IR, GLSA). These constitute future research aims.

\section{References}

1. Blackmon, M., Kitajima, M., Polson, P.: Tool for accurately predicting website navigation problems, non-problems, problem severity, and effectiveness of repairs. In: Proceedings of CHI 2005, pp. 31-40. ACM Press, New York (2005)

2. Card, S., Pirolli, P., Wege, M., Morrison, J., Reeder, R.W., Schraedley, P., Boshart, J.: Information scent as a driver of Web behavior graphs: results of a protocol analysis method for Web usability. In: Proceedings of the CHI 2001, pp. 498-505. ACM Press, New York (2001)

3. Chi, E., Rosien, A., Supattanasiri, G., Williams, A., Royer, C., Chow, C., Robles, E., Dalal, B., Chen, J., Cousins, S.: The Bloodhound Project: Automating Discovery of Web Usability Issues using the InfoScent Simulator. In: Proceedings CHI 2003, pp. 505-512. ACM Press, New York (2003) 
4. Habuchi, Y., Kitajima, M., Takeuchi, H.: Comparison of eye movements in searching for easy-to-find and hard-to-find information in a hierarchically organized information structure. In: Proceedings of ETRA 2008, Savannah, Georgia, pp. 131-134. ACM Press, New York (2008)

5. Katsanos, C., Tselios, N., Avouris, N.: InfoScent evaluator: a semi-automated tool to evaluate semantic appropriateness of hyperlinks in a web site. In: Proceedings of OZCHI 2006, Sydney, Australia, pp. 373-376. ACM Press, New York (2006)

6. Landauer, T.K., Dumais, S.: A solution to Plato's problem: The latent semantic analysis theory of acquisition, induction, and representation of knowledge. Psychological Review 104(2), 211-240 (1997)

7. Pirolli, P.: Information Foraging Theory: Adaptive Interaction with Information. Oxford University Press, USA (2007)

8. Miller, C.S., Remington, R.W.: Modeling information navigation: implications for information architecture. Human-Computer Interaction 19(3), 225-271 (2004) 